\title{
Occult intrasacral meningocoele
}

\author{
ROMA A. JOSEPH AND THOMAS McKENZIE
}

From the Neurosurgical Department, General Hospital, San Fernando, Trinidad, W.I.

\begin{abstract}
SUMMARY A case is reported of the rare lesion occult intrasacral meningocoele in a 27-year-old woman who developed symptoms for the first time shortly after the birth of her fourth child. The terminology of the condition is discussed and its pathogenesis, mode of presentation, and treatment reviewed.
\end{abstract}

The term 'occult intrasacral meningocoele' first used by Enderle (1932) describes the condition of abnormal dilatation of the meninges within the confines of the sacral spinal canal. Various workers have pointed out that this term is not strictly accurate in that a meningocoele implies a hernial protrusion of the meninges through a defect in the skull or vertebral column. Thus, Howieson, Norrell, and Wilson (1968) prefer to speak of 'expansion of the subarachnoid space in the lumbosacral region'. In two of their cases the expanded thecal sac terminated at the normal level, with multiple neurofibromatosis as an associated feature. It would seem better to reserve the description of Howieson and colleagues for these latter cases. The prolongation of the subarachnoid space beyond the normal level is probably better described as a caudal diverticulum of the thecal sac. In practice, there is much in favour of retaining the designation 'occult intrasacral meningocoele' if the original idea of an abnormal prolongation of the meninges within the sacral spinal canal is respected. This would remove the confusion in nomenclature caused by the inclusion of such entities as perineural cysts, meningeal cysts, spinal extradural cysts, and anterior sacral meningocoele.

\section{CASE REPORT}

A 27-year-old woman of Indian descent was seen in consultation one week after onset of numbness which started in the toes of her left foot and spread progressively upwards to involve the whole of the left lower extremity including the buttock. Her gait had deteriorated to the point where she was unable to walk without help. Later, numbness was noticed in the toes of the right foot. She had no pain or bowel or bladder disturbance. Three months earlier she had given birth to her fourth child and the next day while walking she suddenly developed sharp pain in the lumbar region which radiated into the left buttock and down the left leg and was so severe that she was unable to walk. She was treated conservatively and six weeks later she considered herself to be "back to normal'. At no time did she have bladder or bowel disturbance. Before this event she had no history of pain in her back or leg nor had she any symptoms suggestive of motor, sensory, or sphincter dysfunction. Her pregnancies and deliveries had all been normal.

examination Physical examination showed hypotonia and loss of position sense in the left foot, impairment of pain and touch in the left lower limb from L5 to S4, relative diminution of the left ankle jerk but no actual weakness of the limb. Her gait was slow and uncertain with a slight flail movement of the left foot and some outward rotation of the left leg.

Routine blood examination showed $\mathrm{Hb} 13.3 \mathrm{~g} / 100 \mathrm{ml}$., white cell count $10,000 \mathrm{c} . \mathrm{mm}$, urea $15 \mathrm{mg} / 100 \mathrm{ml}$. Lumbar puncture yielded clear colourless fluid under a pressure of $140 \mathrm{~mm}$ of CSF, lymphocytes $4 / \mathrm{ml}$., polymorphs $1 / \mathrm{ml}$., protein $70 \mathrm{mg} / 100 \mathrm{ml}$., sugar $24 \mathrm{mg} / 100 \mathrm{ml}$., and no manometric block.

RADIOLOGICAL FINDINGS Plain films were passed as normal but, in retrospect, there was mild scalloping of the bodies of the lower sacral vertebrae and the sacral canal was dilated. Myelography showed the thecal sac tapering to its normal termination at the second sacral vertebra, being then prolonged into a bulbous segmented midline sac which occupied the lower end of the sacral canal and bulged to the left (Figs. 1 and 2). In the lateral and oblique projections (Fig. 3) was noted a posteriorly situated radiolucent line which extended into the upper portion of the abnormal sac.

OPERATION The sacral canal was unroofed by a laminectomy and the lamina of the fifth lumbar vertebra was also removed. Within the lower sacral laminae were large vascular channels which bled profusely. The thecal sac extended the entire length of the sacral canal, its lower portion bulging irregularly to the left. Numerous large thin-walled varicose veins were present extradurally on the ventral aspect and left side of the sac. The dura was opened to display a thick cord-like structure which 


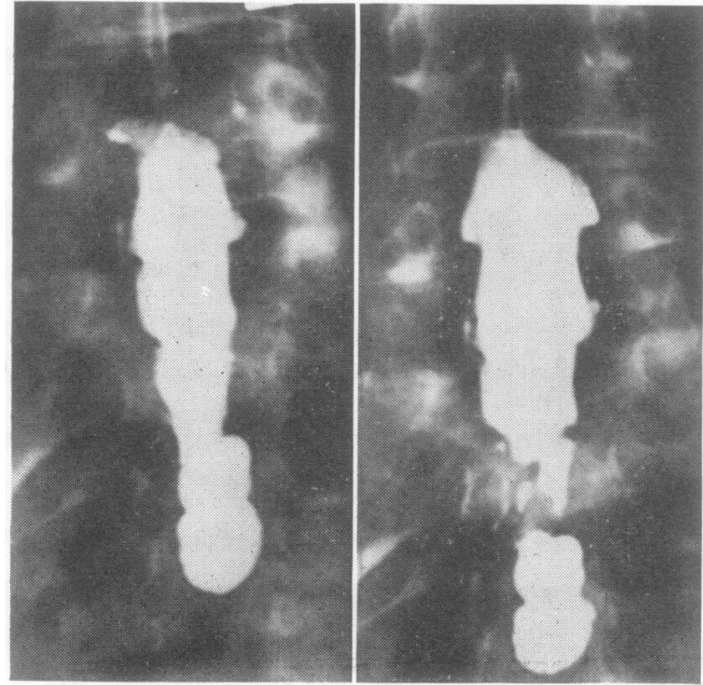

FIG. 1. (Left). Anteroposterior view of lumbosacral theca showing intrasacral meningocoele.

FIG. 2. (Right). Anteroposterior view showing meningocoele isolated with craniad flow of Myodil.

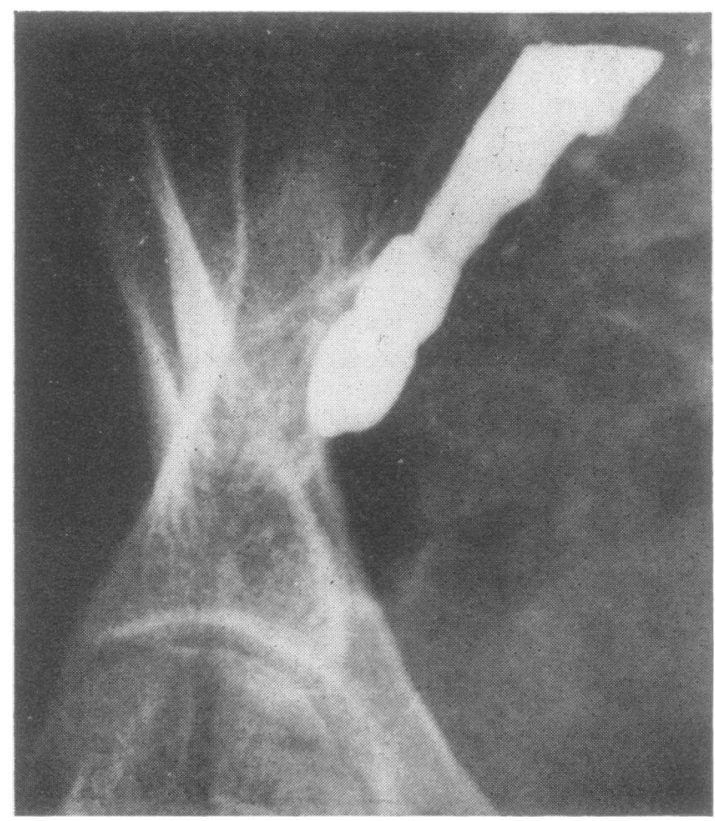

FIG. 3. Oblique view. Note posteriorly-situated linear filling defect in dye column above the lesion.

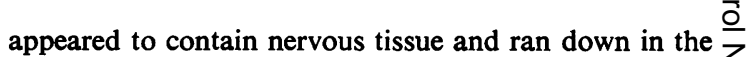
midline into a small terminal lipoma which was firmly $\mathbb{D}$ tethered at the lower end of the canal, at which level the arachnoid was thickened and opaque. The sacral nerve on roots were laterally placed and left the sac at the appropriate levels. The bony decompression already effected was seen to be the only feasible method of dealing with the lesion, so the dura was then closed in the usual $\mathrm{C}$ manner and the wound carefully repaired in layers.

Post-operatively there was rapid regression of abnormal physical signs and by the fourth day numbness was limited to the left foot only. Position sense was now normal. Three weeks after operation she was walking normally and, while there was mild subjective numbness of both feet, neurological examination showed no actual motor or sensory deficit.

\section{DISCUSSION}

PATHOGENESIS Most theories would seem to place $\vec{\circ}$ occult intrasacral meningocoeles somewhere in the $\vec{A}$ broad group of anomalies that include spina bifida ${ }_{\sigma}$ occulta on the one hand and complete craniorachischisis on the other. The evidence suggests that there are different types of occult intrasacral meningocoeles $\omega_{\omega}$ and that different causative factors may be present ip $A$ individual cases. Enderle (1932) suggested failure of ascent of the meningeal sac as a possible explanatio $\vec{D}, \omega$ recalling that in the foetus the neural tube and durfe sac extend beyond the coccyx to the filum caudafe and that the neural tube later retracts upwards, maintaining continuity with its former site of tes-e mination by means of the filum terminale, around क which in turn the dural sac constricts to produce the normal adult pattern.

Other workers have put forward attractive hypotheses in favour of the idea that increased cerebro-o spinal fluid pressure resulting from disturbances of the cerebrospinal fluid mechanism may be responsible for spina bifida and anencephaly and related $\frac{\square}{\varnothing}$ defects. Gardner (1966) has pointed out that hydro- $\varrho$ cephalomyelia exists as a physiological state in the $\overrightarrow{\vec{O}}$ normal embryo, is compensated by leakage of fluid 3 into the developing subarachnoid space, and that interference with this process would lead to failure of narrowing of the central canal of the cord, the height of the internal pressure reached determining the particular anomaly that results, from a minor skeletal 0 defect through syringomyelia and myelocoele to total rachischisis.

Experimental work has shown that proper neural induction is dependent upon normal development of $\circ$ mesoderm (Campbell, 1948). Thus the range of congenital anomalies of which occult intrasacralo meningocoeles are taken to be a part may conceivably result from varying degrees of mesenchymalo defect in the embryo. More to the point is the clinical 0 association of neurofibromatosis with occult intra- 
sacral meningocoele. Howieson et al. (1968) suggest that in these cases a primary mesodermal defect involving either the vertebral bodies or the dura mater or both leads to 'expansion of the subarachnoid space', as the cerebrospinal fluid pulsations erode the dysplastic vertebral bodies or stretch the weakened dura. The idea has frequently been expressed that some cases of expansion of the subarachnoid space in the spinal canal including occult intrasacral meningocoele may well represent formes frustes of Von Recklinghausen's disease or other heritable disorders of connective tissue like Marfan's syndrome and the Ehlers-Danlos syndrome.

MODE OF PRESENTATION Cases have been reported at all ages ranging from early childhood to the eighth decade, often with a history of chronic bladder and bowel dysfunction going back many years before eventual diagnosis. A study of the reports indicates a predominance of females but there is no evidence to suggest that this is due to anything but coincidence. A hereditary element is supported by the occasional presence of related congenital defects like spina bifida occulta and congenital hemivertebrae in close relatives but no two cases of the condition have been described in the same family.

One of the commonest symptoms is pain, either localized in the back or referred down the legs, being either intermittent in character or of progressive severity. In a few instances the pain has been relieved by lying down and increased on coughing, laughing, or straining, this being thought to be due to changes in the degree of filling of the sac in these circumstances. Sometimes trauma has been the precipitating factor, and Pool (1952) has reported a similar instance like the present where the condition became manifest immediately after childbirth. It is tempting to speculate about a 'blow-out' effect attendant on the intense straining associated with the process of childbirth causing the arachnoid to herniate through a possible dural defect. An almost equally common finding has been sphincter dysfunction which takes the form of dysuria, retention, constipation, or incontinence of bowel orbladder and, less obviously, recurrent urinary tract infection. Sexual impotence is an occasional finding. A proportion of patients display stigmata like the cutaneous lesions of multiple neurofibromatosis, skin nodules, sacral lipomata, dermal sinus, sacral hypertrichosis or pigmentation, and, as in Walker's (1944) case, an atrophic leg. Examination findings in the spine may be limited to tenderness over the lumbosacral spines and in the paravertebral region. The characteristic sensory deficit takes the form of altered sensation or paraesthesiae in the saddle area but the legs may be similarly involved in varying degree. Frank motor involvement is rather less common but weakness of the lower extremity may be encountered. The ankle reflex may be weak or absent and the anal sphincter may show loss of tone.

Plain radiographs may reveal no gross abnormality but common findings are widening of the sacral canal with scalloping of the vertebral bodies (Mitchell, Lourie, and Berne, 1967), spina bifida occulta, increase in the size of the sacral hiatus, and lumbarization of the first sacral vertebra. Tomography may be necessary to show the presence of a widened sacral canal. The diagnosis of occult intrasacral meningocoele is definitively established at myelography. The contrast-filled expansion of the subarachnoid space is shown within the lower sacral canal, often linked by a narrow isthmus to the normal termination of the thecal sac at the level of the second sacral vertebra. The abnormal sac sometimes shows a segmented appearance which, in some cases at least, appears to correspond with the individual sacral segments opposite which it lies. Enderle's original case demonstrated the need in some instances to repeat the myelogram after a lapse of 24 to 48 hours to bring to light the condition.

The differential diagnosis includes intradural and extradural tumours, inflammatory disease, intervertebral disc lesions, disseminated sclerosis, and residual effects of poliomyelitis.

TREATMENT Pool (1952) has pointed out the need for prompt surgical interference once the diagnosis is made, before permanent nerve-root or spinal cord damage results. There are enough cases to show the beneficial effect of surgery in relieving the adverse clinical features associated with this condition. It may be possible to ligate the abnormal sac at the isthmus which is often present at the normal level of the thecal cul-de-sac, but decompression of the lesion by de-roofing the sacral canal may be all that is possible. Of interest is the terminal intradural lipoma which was present in the case now reported, and Pool (1952) and Walker (1944) have described a similar finding in one of their cases. Microscopic examination of the meningocoele sac may show its derivation from the meninges or, as in Palazzoli's (1963) case, from the arachnoid alone, but often its wall may be seen to be composed merely of fibrous tissue.

\section{REFERENCES}

Enderle, C. (1932). Meningocoele intrasacrale occulto (rivelato con la mielografia). Riv. Neurol., 5, 418-423.

Campbell, J. B. (1948). Congenital anomalies of the neural axis: surgical management based on embryologic considerations. Amer. J. Surg., 75, 231-256. 
Gardner, W. J. (1966). Embryologic origin of spinal malformations. Acta. radiol. Diagn., 5, 1013-1023.

Howieson, J., Norrell, H. A., and Wilson, C. B. (1968). Expansion of the subarachnoid space in the lumbosacral region. Radiology, 90, 488-492.

Mitchell, G. E., Lourie, H., and Berne, A. S. (1967). The various causes of scalloped vertebrae with notes on their pathogenesis. Radiology, 89, 67-74.

Palazzoli, A. (1963). Rilievi clinici e patogenetici su un caso di meningocele sacrale occulto simulante un'ernia discale. Bol. Soc. med-chir., 17, 203-225.
Pool, J. L. (1952). Spinal cord and local signs secondary to occult sacral meningoceles in adults. Bull. N.Y. Acad. Med., 28, 655-663.

Shapiro, R. (1962). Myelography. Year Book Medical Publishers: Chicago.

Walker, A. E. (1944). Dilatation of vertebral canal associated with congenital anomalies of spinal cord. Amer. J. Roentgenol., 52, 571-582. 